\title{
Brahma-related gene 1-associated expression of 9-27 and IFI-27 is involved in acquired cisplatin resistance of gastric cancer cells
}

\author{
HYE RAN LEE ${ }^{1}$, HYUN KYUNG NO ${ }^{2}$, CHUN JEIH RYU ${ }^{3}$ and HYUN-JU PARK ${ }^{2}$ \\ ${ }^{1}$ Department of Internal Medicine, Inje University Ilsan Paik Hospital, Goyang, Gyeonggi-do 411-706; \\ ${ }^{2}$ School of Pharmacy, Sungkyunkwan University, Suwon, Gyeonggi-do 440-746; \\ ${ }^{3}$ Department of Bioscience and Biotechnology, Sejong University, Kwanjin-Gu, Seoul 143-747, Republic of Korea
}

Received March 6, 2013; Accepted June 24, 2013

DOI: $10.3892 / \mathrm{mmr} .2013 .1576$

\begin{abstract}
In order to investigate the mechanism of cisplatin resistance, a cisplatin-resistant human gastric cancer cell line was established. Subsequent to the exposure of the YCC-3 gastric cancer cell line to equal concentrations of cis-diammine-dichloroplatinum (II) (cisplatin, CDDP) for 6 months, a cisplatin-resistant cell line was established (YCC-3/R). To determine the molecular mechanism of cisplatin resistance in YCC-3/R cells, differentially expressed genes (DEGs) were investigated between $\mathrm{YCC}-3$ and $\mathrm{YCC}-3 / \mathrm{R}$ by annealing control primer-based reverse transcriptase-polymerase chain reaction (ACP RT-PCR) technology. Eleven DEGs were successfully identified and sequenced. Among them, interferon-induced transmembrane protein 1 (9-27) and interferon $\alpha$-inducible protein 27 (IFI-27) were markedly increased in YCC-3/R cells. In addition, western blot analysis demonstrated that the Brahma-related gene 1 (BRG1), which was observed to selectively activate 9-27 and IFI-27 genes, was overexpressed in YCC-3/R cells. The results suggested that the BRG1-associated expression of 9-27 and IFI-27 is involved in cisplatin resistance in gastric cancer cells.
\end{abstract}

\section{Introduction}

Gastric cancer remains a leading cause of mortality in certain countries. Although the occurrence rate of gastric cancer has been on the decrease worldwide, certain Asian countries (including Korea and Japan), and certain European and South American countries still exhibit a high incidence rate (1). As is the case with other types of cancer, early-stage gastric cancer may be treated by surgery. However, a large number of patients are already in advanced stages at the time of diagnosis, and thus

Correspondence to: Professor Hyun-Ju Park, School of Pharmacy, Sungkyunkwan University, 300 Cheoncheon-dong, Suwon, Gyeonggi-do 440-746, Republic of Korea

E-mail: hyunju85@skku.edu

Key words: gastric cancer, cisplatin resistance, 9-27, IFI-27, Brahma-related gene 1 have a worse prognosis. Although numerous chemotherapeutic agents have been used to treat gastric cancer patients, one of the predominant obstacles in treating these patients is chemoresistance. Cisplatin is the most frequently used drug for the treatment of patients with advanced gastric cancer. However, gastric cancer cells may be inherently insensitive to cisplatin and acquire resistance against cisplatin during treatment (2). Acquired drug resistance is suggested to be multifactorial, involving host factors and numerous genetic and molecular events (3). Studies have demonstrated that resistance may be related to decreased drug accumulation, alteration of intracellular drug distribution, changes in drug-target interaction, cell-cycle deregulation, increased damaged-DNA repair and reduced apoptotic response (4-12). Therefore, it is important to determine the mechanism of resistance to cisplatin.

Annealing control primer-based-reverse transcriptase-polymerase chain reaction (ACP RT-PCR) technology regulated by an annealing control primer has been used to identify differentially expressed genes in several tissues including cancer tissues (13-15). This method specifically targets sequence hybridization to the template via a polydeoxyinosine (poly dI) linker. The ACP-based PCR system may be facilitated to identify differentially expressed genes (DEGs) from samples exhibiting low mRNA levels possibly without generating false positives. Therefore, using this technique, it was possible to search for related genes that may be responsible for cisplatin resistance.

This study aimed to determine the correlation between gene expression changes and cisplatin-resistance in gastric cancer cells. In our previous study, a cisplatin-resistant gastric cancer cell line (YCC-3/R) was established by chronic exposure of a human gastric carcinoma cell line (YCC-3) to cisplatin, and it was observed that p27 expression was upregulated in the YCC-3/R cells (16). In order to identify other genes that may be responsible for resistance to cisplatin, in the present study, DEGs between YCC-3 and YCC-3/R cells were investigated using ACP-based RT-PCR technology. It was demonstrated that interferon-induced transmembrane protein 1 (9-27) and interferon $\alpha$-inducible protein 27 (IFI-27) genes were highly expressed in YCC-3/R cells. Subsequent studies suggested that the Brahma-related gene 1 (BRG1)-associated expression of 9-27 and IFI-27 genes may be involved in resistance to cisplatin in YCC-3/R cells. 
Table I. List of genes differentially expressed in YCC-3/R.

\begin{tabular}{|c|c|c|}
\hline DEG no. & GeneBank accession no. & Sequence homology search \\
\hline DEG1 ${ }^{\text {a }}$ & BC15492 & Homo sapiens interferon, $\alpha$-inducible protein 27 (IFI-27) \\
\hline DEG4 ${ }^{\mathrm{a}}$ & BC000897 & Homo sapiens interferon induced transmembrane protein 1 (9-27) (IFITM1) \\
\hline DEG5 $^{\mathrm{a}}$ & ВC000897 & Homo sapiens interferon induced transmembrane protein 1 (9-27) (IFITM1) \\
\hline DEG7 & ВC000027 & Homo sapiens transmembrane emp24 protein transport domain containing 3 \\
\hline DEG9 $9^{a}$ & ВC000897 & Homo sapiens interferon induced transmembrane protein 1 (9-27) (IFITM1) \\
\hline DEG14 & ВC015592 & Homo sapiens deltex 4 homolog (Drosophilla) \\
\hline DEG15 ${ }^{\mathrm{a}}$ & $\begin{array}{l}\text { AV73350 (EST) } \\
\text { AP008920 }\end{array}$ & $\begin{array}{l}\text { Homo sapiens } \mathrm{cDNA} \text { clone } \\
\text { Homo sapiens mitochondrial DNA, complete genome }\end{array}$ \\
\hline DEG18 ${ }^{\mathrm{a}}$ & AY244097 & Homo sapiens isolate Mongolian 259 mitochondrial control region \\
\hline DEG19 & ВC070194 & Homo sapiens ribosomal protein, large, $\mathrm{P} 0$ \\
\hline DEG20 & AL110297 & Homo sapiens mRNA \\
\hline DEG21 ${ }^{\mathrm{a}}$ & BC012355 & Homo sapiens solute carrier family 6 (neurotransmitter transporter, creatine) \\
\hline
\end{tabular}

${ }^{\mathrm{a} O v e r e x p r e s s i o n ~ i n ~ Y C C-3 / R . ~ D E G 13 ~ s e q u e n c i n g ~ f a i l e d . ~}$

\section{Materials and methods}

Cell lines and culture conditions. YCC-3 (donated by Professor Hyun-Chul Chung, Yonsei Cancer Center, Yonsei University, Seoul) a human gastric cancer cell line, was cultured with Dulbecco's modified Eagle's medium (DMEM) (Invitrogen Life Technologies, Carlsbad, CA, USA) supplemented with $10 \%$ heat-inactivated fetal bovine serum (Hyclone Laborotories Inc., Logan, UT, USA), $3.7 \mathrm{mg} / \mathrm{ml}$ sodium bicarbonate, $2 \mathrm{mM} \mathrm{L}$-glutamine, $25 \mathrm{mM}$ HEPES and $1 \%$ penicillin/streptomycin. The culture was maintained under a humidified $5 \% \mathrm{CO}_{2}$ atmosphere at $37^{\circ} \mathrm{C}$. The culture was passaged twice or three times a week. YCC-3/R was established by chronic exposure to equal concentrations $(0.5 \mu \mathrm{g} / \mathrm{ml})$ of cisplatin for 6 months as described previously (16).

ACP RT-PCR analysis. Total RNA extracted from YCC-3 and YCC-3/R cells was used for the synthesis of first-strand cDNA by reverse transcriptase. Reverse transcription was performed for $1.5 \mathrm{~h}$ at $42^{\circ} \mathrm{C}$ in a final reaction volume of $20 \mu \mathrm{l}$ containing $3 \mu \mathrm{g}$ of the purified total RNAs, $4 \mu 15 \mathrm{X}$ reaction buffer (Promega, Madison, WI, USA), $5 \mu \mathrm{l}$ dNTPs (each $2 \mathrm{mM}$ ), $2 \mu \mathrm{l} 10 \mu \mathrm{M}$ dT-ACP1 [5'-CGTGAATGCTGCGACTACGATIIIIIT(18)-3', Seegene Inc., Seoul, Korea], $0.5 \mu 1$ RNasin ${ }^{\circledR}$ RNase Inhibitor (40 U/ $\mu 1$; Promega) and $1 \mu \mathrm{l}$ Moloney murine leukemia virus reverse transcriptase (200 U/ $\mu 1$; Promega). First-strand cDNA was diluted by the addition of $80 \mu 1$ ultra-purified water for the GeneFishing $^{\mathrm{TM}}$ PCR (Seegene Inc., Seoul, Korea), and stored at $-20^{\circ} \mathrm{C}$ until use. Differentially expressed genes were screened by the ACP-based PCR method using the GeneFishing DEG kits (Seegene Inc.) (13). Briefly, second-strand cDNA synthesis was conducted at $50^{\circ} \mathrm{C}$ during one cycle of first-stage PCR in a final reaction volume of $20 \mu \mathrm{l}$ containing 3-5 $\mu \mathrm{l}(\sim 50 \mathrm{ng})$ diluted first-strand cDNA, $1 \mu \mathrm{l}$ dT-ACP2 $(10 \mu \mathrm{M}), 1 \mu 110 \mu \mathrm{M}$ arbitrary ACP, and $10 \mu \mathrm{l} 2 \mathrm{X}$ Master Mix (Seegene Inc.). The PCR protocol for second-strand synthesis was one cycle at $94^{\circ} \mathrm{C}$ for $1 \mathrm{~min}$, followed by $50^{\circ} \mathrm{C}$ for $3 \mathrm{~min}$, and $72^{\circ} \mathrm{C}$ for $1 \mathrm{~min}$. When the second-strand DNA synthesis was completed, the second-stage PCR amplification protocol was 40 cycles of $94^{\circ} \mathrm{C}$ for $40 \mathrm{sec}$, followed by $65^{\circ} \mathrm{C}$ for $40 \mathrm{sec}, 72^{\circ} \mathrm{C}$ for $40 \mathrm{sec}$ and a $5 \mathrm{~min}$ final extension at $72^{\circ} \mathrm{C}$. The amplified PCR products were separated on $2 \%$ agarose gel stained with ethidium bromide. The differentially expressed bands were re-amplified and extracted from the gel using the GENCLEAN ${ }^{\circledR}$ II kit (Q-BIO Gene, Carlsbad, CA, USA) and directly sequenced with ABI PRISM ${ }^{\circledR}$ 3100-Avant Genetic Analyzer (Applied Biosystems, Foster City, CA, USA). The identity of each product was confirmed by sequence homology analysis using the Basic Local Alignment Search Tool (BLAST) (17).

Western blot analysis. Cell lysates were boiled in Laemmli sample buffer for $5 \mathrm{~min}$ at $95^{\circ} \mathrm{C}$ and $30 \mu \mathrm{g}$ protein lysates from each sample were electrophoresed on a $12 \%$ sodium dodecyl sulphate-polyacrylamide gel and then transferred to a polyvinylidine fluoride membrane (Bio-Rad, Hercules, CA, USA) and electrophoresed at a constant $40 \mathrm{~V}$ for $90 \mathrm{~min}$. Western blot analysis was conducted as described previously (18). The membrane was pre-blocked for $1 \mathrm{~h}$ at room temperature (RT) in Tris-buffered saline Tween-20 (TBST) containing 5\% skimmed milk. The blot was incubated for $3 \mathrm{~h}$ at RT with anti-BRG1 antibody (Amersham Biosciences, Seoul, Korea) and $\beta$-actin (Abcam, Cambridge, UK) in TBST. The blot was then washed three times for 10 min with TBST. The membrane was incubated for $1 \mathrm{~h}$ with peroxidase-linked species-specific whole antibodies. Protein was detected using an enhanced chemiluminescence western blot analysis system (Amersham Biosciences).

\section{Results}

To screen DEGs between YCC-3 and YCC-3/R cells, mRNAs from the two types of cells were extracted and subjected to ACP RT-PCR analysis using 20 arbitrary ACPs provided by Seegene Inc. (13-15). As a result, 21 bands appeared to be differentially expressed between YCC-3 and YCC-3/R cells, and 11 bands appeared to be upregulated in YCC-3 cells (Fig. 1, white arrows), while 10 bands appeared to be upregulated in 

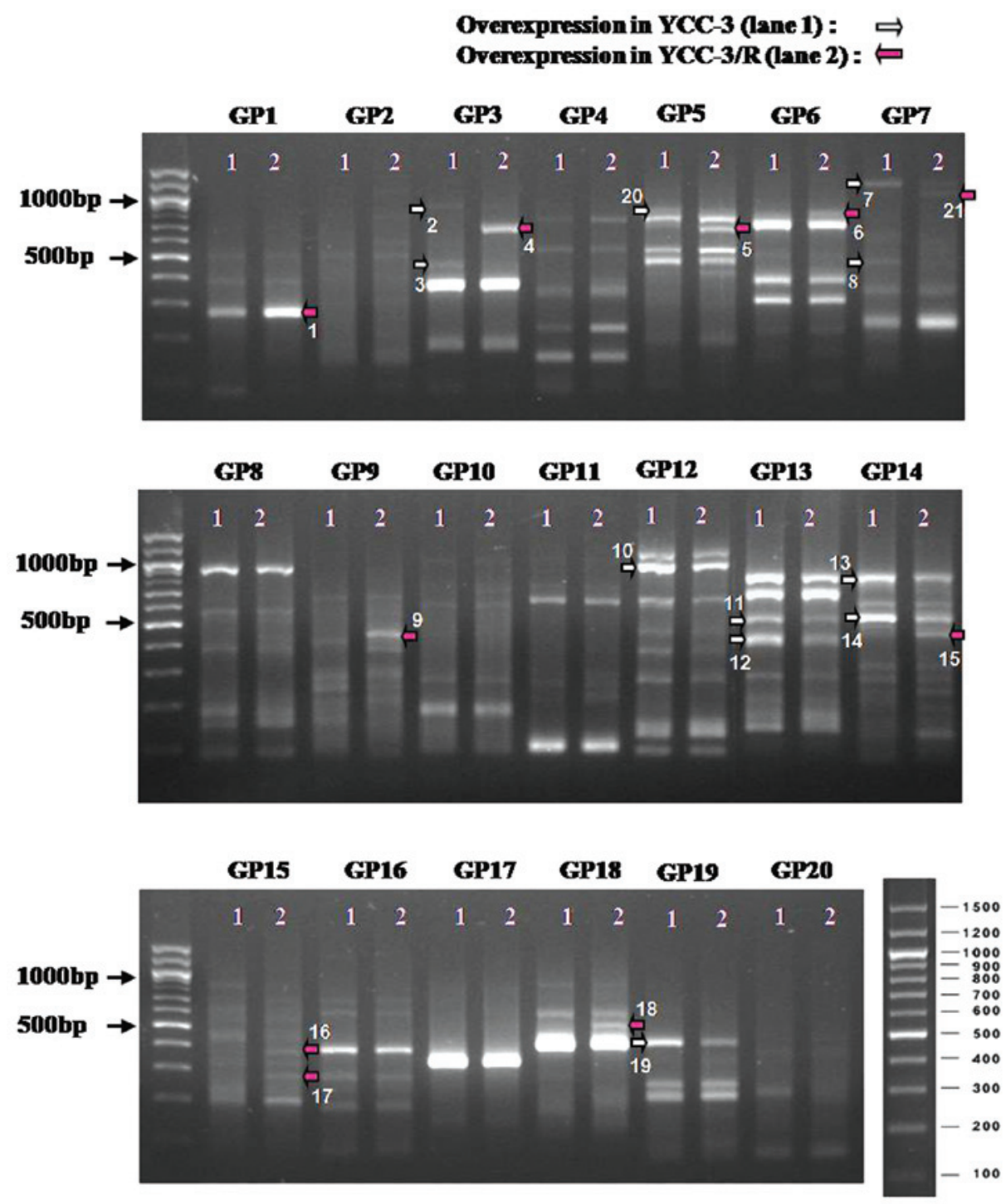

Figure 1. Annealing control primer-based reverse transcriptase-polymerase chain reaction (ACP RT-PCR) analysis for the identification of differentially expressed genes (DEGs) between YCC-3 and YCC-3/R cells. Agarose gel photograms (2\%) show DEGs obtained from YCC-3 (lane 1) and YCC-3/R (lane 2) cells by using 20 arbitrary ACPs. The white arrows indicate the DEGs that show a higher expression in YCC-3 cells, while the red arrows indicate the DEGs that show a higher expression in YCC-3/R cells.

YCC-3/R cells (Fig. 1, red arrows). DNA bands were excised and sequenced for further analysis. However, only 11 DNA fragments including DEG1, 4, 5, 7, 9, 14, 15, 18, 19, 20 and 21 were successfully sequenced (Table I). A sequence homology search using BLAST revealed that the bands were IFI-27, 9-27, transmembrane emp24 protein transport domain containing 3 , deltex 4 homolog, unknown cDNA clone, mitochondrial DNA, Mongolian 259 mitochondrial control region, and ribosomal protein. Seven DEGs were upregulated in YCC-3/R cells while 4 DEGs were downregulated. Notably, three out of seven upregulated DEGs in YCC-3/R were interferon $\alpha$-inducible gene 9-27 and 1 DEG was another interferon $\alpha$-inducible gene IFI-27.

Interferon $\alpha$-inducible genes $9-27$ and $I F I-27$ were investigated as a previous study suggested that BRG1, a key component of the mammalian ATP-dependent chromatin-remodeling SW12-SNF2 complex, selectively activates a subset of interferon- $\alpha$-inducible genes including 9-27 and IFI-27 (19). Therefore, BRG1 expression was investigated by western blot

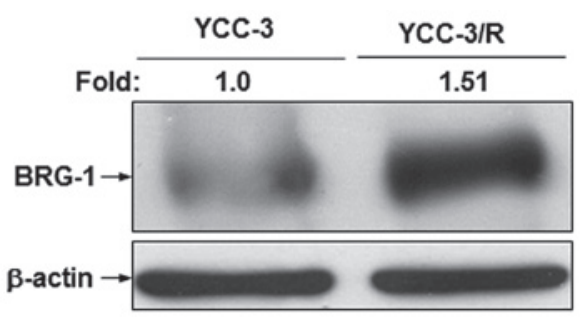

Figure 2. Western blot analysis of Brahma-related gene 1 (BRG1) protein in YCC- 3 and YCC-3/R cells. $\beta$-actin was used as a reference for gene expression. The intensity of each western blot signal was quantified by Image $\mathrm{J}$.

analysis to identify whether BRG1 expression is associated with the increased expression of 9-27 and IFI-27 (Fig. 2). As expected, the BRG1 protein was upregulated in YCC-3/R cells compared with that in the YCC-3 cells, suggesting that the BRG1-associated expression of 9-27 and IFI-27 is involved in acquired cisplatin resistance of gastric cancer cells. 


\section{Discussion}

In our previous study, a cisplatin-resistant gastric cancer cell line (YCC-3/R) was established by the chronic exposure of a human gastric carcinoma cell line (YCC-3) to cisplatin (16). To screen genes that may be related to cisplatin resistance in YCC-3/R, ACP RT-PCR technology was used as conventional display methods are labor intensive and lead to a high degree of false positivity. The basis of ACP technology is the unique tripartite structure of a specific oligonucleotide primer (ACP), which contains distinct 3'- and 5'-end regions separated by a regulator and the interactions of each portion of this primer during two-stage PCR (13-15). ACP RT-PCR technology yielded 11 novel DEGs and among these IFI-27 and 9-27 were particularly noteworthy (Fig. 1). IFI-27 and 9-27 are known as interferon-inducible proteins that are involved in the control of cell growth. The 9-27 gene is suspected to encode a protein that associates with other membrane proteins, forming a multimeric complex involved in the transduction of anti-proliferative and homotypic adhesion signals (20). In addition, 9-27 was shown to be correlated with susceptibility to natural killer cells and their invasiveness in gastric cancer cells (21). Luker et al (22) also showed that a paclitaxel-resistant cell line overexpresses IFN-inducible p27 (IFI-27), and confers resistance to paclitaxel in breast cancer cells. A previous study suggested that 9-27 arrests cell cycle progression in the $\mathrm{G} 1$ phase (23). In a previous study, p27-dependent cell cycle arrest was demonstrated to be involved in acquired cisplatin resistance of YCC-3/R cells (16). Therefore, it may be speculated that the induction of 9-27 and IFI-27 contributes to acquired cisplatin resistance of YCC-3/R cells.

BRG1 is a predominant component of the mammalian ATP-dependent chromatin-remodeling SW12/SNF2 complex and interacts with signal transducer and activator of transcription (STAT2). Notably, BRG1 overexpression is associated with advanced-stage human gastric carcinomas (24). Subsequent studies demonstrated that BRG1 selectively activated a subset of interferon- $\alpha$-inducible genes including 9-27 and IFI-27 (19). A recent study also demonstrated that the downregulation of BRG1 and brahma (Brm) results in enhanced cell sensitivity to cisplatin (23). According to these studies, incomplete repair of cisplatin intrastrand adducts, interstrand crosslinks and interstrand crosslinks-induced DNA double-strand breaks are observed in BRG1- and Brm-depleted cells. BRG1 and Brm deficiency also results in impaired chromatin relaxation, altered check point activation and enhanced apoptosis (25). Thus, BRG1 and Brm may modulate cisplatin cytotoxicity via chromatin remodeling and facilitate the DNA repair factors following DNA damage recognition and sensitivity to UV radiation (25). In conclusion, an increased expression of BRG1 and BRG1-inducible genes, 9-27 and IFI-27, may contribute to acquired resistance to cisplatin in gastric cancer cells.

\section{Acknowledgements}

The authors would like to thank Professor Hyun-Chul Chung (Yonsei Cancer Center, Yonsei University, Seoul) for donating the YCC-3 cell line. This study was funded by the Samsung Research Fund, Sungkyunkwan University, 2006, and the Inje University Research Grant in 2005.

\section{References}

1. Kelley JR and Duggan JM: Gastric cancer epidemiology and risk factors. J Clin Epidemiol 56: 1-9, 2003.

2. Banerjee D, Mayer-Kuckuk P, Capiaux G, Budak-Alpdogan T, Gorlick R and Bertino JR: Novel aspects of resistance to drugs targeted to dihydrofolate reductase and thymidylate synthase. Biochim Biophys Acta 1587: 164-173, 2002.

3. Gottesman MM: Mechanisms of cancer drug resistance. Annu Rev Med 53: 615-627, 2002.

4. Larsen AK, Escargueil AE and Skladanowski A: Resistance mechanisms associated with altered intracellular distribution of anticancer agents. Pharmacol Ther 85: 217-229, 2000.

5. Fuertes MA, Alonso C and Pérez JM: Biochemical modulation of Cisplatin mechanisms of action: enhancement of antitumor activity and circumvention of drug resistance. Chem Rev 103: 645-662, 2003.

6. Konishi H, Usui T, Sawada H, Uchino H and Kidani Y: Effects of anticancer platinum compounds on Escherichia coli strains with normal and defective DNA repair capacity. Gann 72: 627-630, 1981.

7. Beck DJ, Popoff S, Sancar A and Rupp WD: Reactions of the UVRABC excision nuclease with DNA damaged by diamminedichloroplatinum(II). Nucleic Acids Res 13: 7395-7412, 1985.

8. Fram RJ, Cusick PS and Marinus MG: Studies on mutagenesis and repair induced by platinum analogs. Mutat Res 173: 13-18, 1986.

9. Kartalou M and Essigmann JM: Mechanisms of resistance to cisplatin. Mutat Res 478: 23-43, 2001.

10. Brown R, Hirst GL, Gallagher WM, et al: hMLH1 expression and cellular responses of ovarian tumour cells to treatment with cytotoxic anticancer agents. Oncogene 15: 45-52, 1997.

11. Fink D, Nebel S, Aebi S, et al: The role of DNA mismatch repair in platinum drug resistance. Cancer Res 56: 4881-4886, 1996.

12. Vaisman A, Varchenko M, Umar A, et al: The role of hMLH1, hMSH3, and hMSH6 defects in cisplatin and oxaliplatin resistance: correlation with replicative bypass of platinum-DNA adducts. Cancer Res 58: 3579-3585, 1998.

13. Kim YJ, Kwak CI, Gu YY, Hwang IT and Chun JY: Annealing control primer system for identification of differentially expressed genes on agarose gels. Biotechniques 36: 424-426, 2004.

14. Hwang KC, Cui XS, Park SP, et al: Identification of differentially regulated genes in bovine blastocysts using an annealing control primer system. Mol Reprod Dev 69: 43-51, 2004.

15. Lee KY, Huang SM, Li S and Kim JM: Identification of differentially expressed genes in papillary thyroid cancers. Yonsei Med J 50: 60-67, 2009.

16. Le TV, Seo Y, Ryu CJ, Lee HR and Park HJ: Increased expression of p27 is associated with the cisplatin resistance in gastric cancer cell line YCC-3. Arch Pharm Res 33: 1127-1132, 2010.

17. Altschul SF, Gish W, Miller W, Myers EW and Lipman DJ: Basic local alignment search tool. J Mol Biol 215: 403-410, 1990.

18. Yoon IS, Chung JH, Hahm SH, et al: Ribosomal protein S3 is phosphorylated by Cdk1/cdc2 during G2/M phase. BMB Rep 44: 529-534, 2011.

19. Huang M, Qian F, Hu Y, Ang C, Li Z and Wen Z: Chromatinremodelling factor BRG1 selectively activates a subset of interferon-alpha-inducible genes. Nat Cell Biol 4: 774-781, 2002.

20. Deblandre GA, Marinx OP, Evans SS, et al: Expression cloning of an interferon-inducible $17-\mathrm{kDa}$ membrane protein implicated in the control of cell growth. J Biol Chem 270: 23860-23866, 1995.

21. Kang HC, Kim IJ, Park JH, et al: Identification of genes with differential expression in acquired drug-resistant gastric cancer cells using high-density oligonucleotide microarrays. Clin Cancer Res 10: 272-284, 2004.

22. Luker KE, Pica CM, Schreiber RD and Piwnica-Worms D: Overexpression of IRF9 confers resistance to antimicrotubule agents in breast cancer cells. Cancer Res 61: 6540-6547, 2001.

23. Yang G, Xu Y, Chen X and Hu G: IFITM1 plays an essential role in the antiproliferative action of interferon-gamma. Oncogene 26: 594-603, 2007.

24. Sentani K, Oue N, Kondo H, et al: Increased expression but not genetic alteration of BRG1, a component of the SWI/SNF complex, is associated with the advanced stage of human gastric carcinomas. Pathobiology 69: 315-320, 2001.

25. Kothandapani A, Gopalakrishnan K, Kahali B, Reisman D and Patrick SM: Downregulation of SWI/SNF chromatin remodeling factor subunits modulates cisplatin cytotoxicity. Exp Cell Res 318: 1973-1986, 2012. 